\title{
Immune Optimization of PID Control Parameters for DC Motor
}

\author{
Peng $\mathrm{Li}^{1}$, Yi Shen ${ }^{1,2, *}$, Rong Chen ${ }^{1}$, Rongbing Yan ${ }^{1}$, Mingxin Yuan ${ }^{1,2}$ \\ ${ }^{I}$ (School of Mechanics and Automotive Engineering, Jiangsu University of Science and Technology, \\ Zhangjiagang, China) \\ ${ }^{2}$ (Changzhou Hongmeng Auto Parts Technology Co. Ltd. Changzhou, China)
}

\begin{abstract}
In order to select the optimal PID control parameters for the brushless DC(Direct Current) motor of the two wheeled self balancing electric vehicle, a bacterial foraging algorithm-based polyclonal selection algorithm (BFA-PSA) is proposed. The bacterial foraging algorithm is introduced into the polyclonal selection algorithm (PSA) to solve the convergence phenomenon which often occurs in the basic PSA at the late of the optimization and causes the algorithm to fall into local minima. The optimization results of the functions show that the searching ability and searching efficiency of the proposed BFA-PSA are improved. Finally, on the basis of the transform function model of the brushless DC motor, the proposed algorithm is used in the optimization of the PID control parameters, and the system response results show the validity of the BFA-PSA.
\end{abstract}

Keywords: Brushless DC motor; Bacteria foraging; Clonal selection; PID control

\section{Introduction}

With the development of the economy, the two wheeled self balancing electric vehicle has been one of the travel tools of the people. Fig. 1 gives a famous Segway self balancing electric vehicle. At present, in the balancing vehicle, the brush DC motor is often used. It is no doubt that the driving performance of the brushless DC motor is better than that of the brush DC motor. In order to improve the control effect of the DC motor, the PID control law is very important.

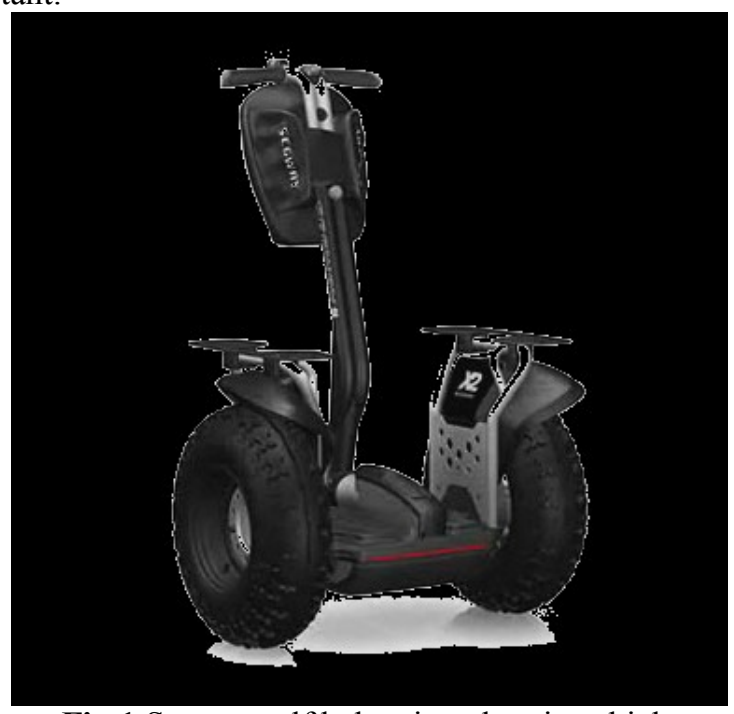

Fig.1 Segway self balancing electric vehicle

In practical engineering, the acquirement of the PID parameters is based on trial and error method, which is not only time-consuming, but the parameters are not very accurate. With the development of the intelligent algorithms, they are widely used in the optimization of the PID parameters and the testing results verify the validity of the optimization of the PID parameters. Zhang [1] introduced the particle swarm optimization algorithm into the PID controller and achieve accurate PID parameters. Yin et al. [2] firstly gives a brief introduction to the ant colony algorithm, and then presents a PID algorithm based ant colony algorithm optimization strategy. Yu et al. [3] realize the PID parameters optimization based on fish swarm algorithm. Aiming at engineering application, Wang et al. [4] put forward a new control strategy of improved variable arguments PID based on the optimization of the immune genetic algorithm. Intelligent algorithms are characterized by global optimization and distributed search; however, the optimization results depend on their optimization ability. In order to further improve the PID control performance, a bacterial foraging algorithmbased polyclonal selection optimization algorithm (BFA-PSA) is proposed in this paper.

* Corresponding author. E-mail: sheny456@hotmail.com 


\section{Transfer Function of Brushless DC Motor}

Taking the two-phase winding conduction as an example, the transfer function of the brushless DC motor [5] can be described as:

$$
G(s)=\frac{\Omega(s)}{U_{d}(s)}=\frac{K_{T}}{L_{a} J s^{2}+\left(r_{a} J+L_{a} B_{v}\right) s+\left(r_{a} B_{v}+k_{e} K_{T}\right)}
$$

Where, $U_{d}$ is the DC bus voltage, $r_{a}$ is the winding resistance, $L_{a}$ is the equivalent inductance of the winding, $K_{e}$ is the line back EMF coefficient, $J$ is the moment of inertia of the rotor, $B_{v}$ is the viscous friction coefficient and $K_{T}$ is the coefficient of the motor torque.

The system construction of the brushless DC motor can be built as shown in Fig.2.

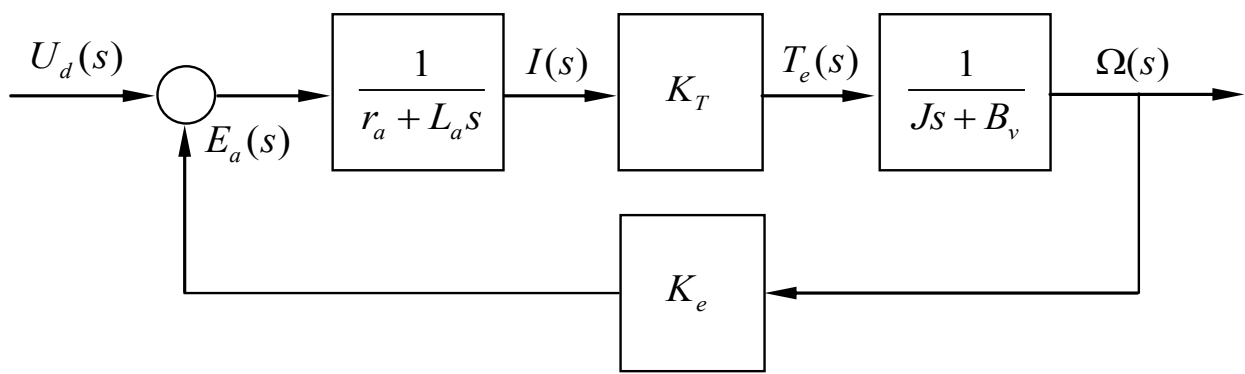

Fig.2 System construction of the brushless DC motor

According to the conventional engineering design approach, the transfer function of the brushless DC motor can be described finally:

$$
\frac{427}{s^{2}+90 s}
$$

Through Eq.(2), the PID control of the brushless DC motor can be realized.

\section{Clonal Selection Optimization Algorithm Based on Bacterial Foraging}

\subsection{Clonal selection algorithm}

The clonal selection algorithm is put forward by Burnet in 1957. Its principle can be described as: Each antibody in population can replicate a certain number of clonal cell according to the affinity between the antibody and the antigen. The number of clonal cells is proportional to the affinity, that is to say, the antibody with higher affinity can replicate more cells. The clonal selection corresponds to the maturation process of the affinity, that is to say, after the replication and mutation operation, the affinity of the individual with low affinity is improved. The antibody with high affinity has small mutation probability; contrarily, the antibody with low affinity has big mutation probability.

The clonal selection algorithm [6] includes clonal operation $T_{c}{ }^{C}$, immune gene operation $T_{g}{ }^{C}$ and clonal selection operation $T_{s}^{C}$. The basic flow can be described as:

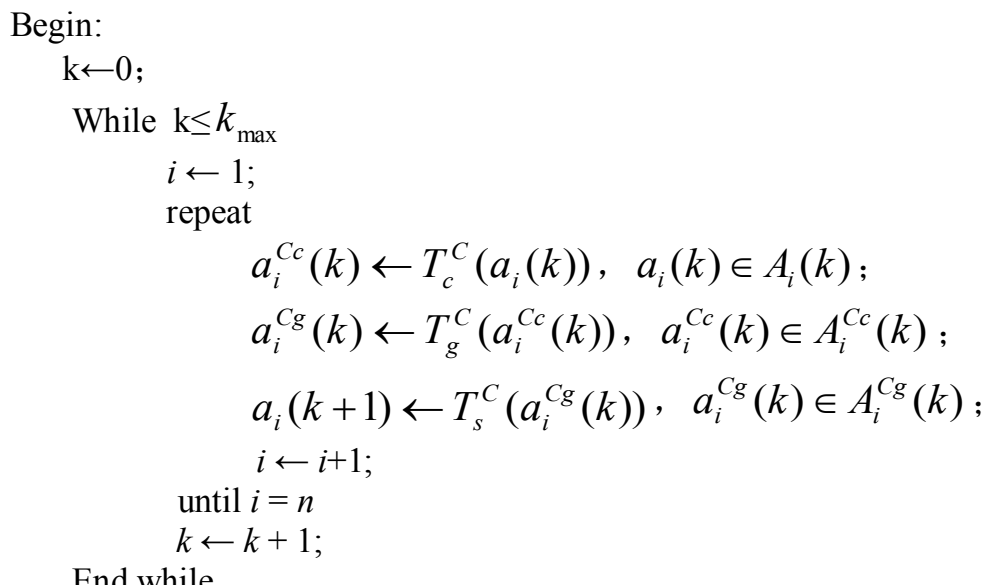




\subsection{Bacterial foraging algorithm}

The bacterial foraging algorithm simulates the foraging behavior of Escherichia coil in the human intestine. The foraging behavior includes the following processes.

(1) Look for the area of the food source.

(2) Judge whether access to the foraging area according to the prior knowledge.

(3) The bacteria will die or move to another suitable foraging area when certain food is consumed or the foraging environments are worse.

According to the above three processes, the bacterial foraging optimization, BFO is put forward in 2002 by K.M. Passino. The BFO is a new bionic swarm intelligence algorithm and includes three operators, namely, chemotaxis operator $T_{c}^{B}$, reproduction operator $T_{r}^{B}$ and elimination-dispersal operator $T_{e}^{B}$. The concrete operation of the three operators is described in [7].

\subsection{Clonal selection algorithm based on bacterial foraging}

The whole flow of clonal selection algorithm based on bacterial foraging can be described as follows:

Begin:

Parameter initialization.

$k \leftarrow 0$;

While $k \leq k_{\max }$

$i \leftarrow 1$;

repeat

$j \leftarrow 1$

repeat

$p_{i}^{B c}(k) \leftarrow T_{c}^{B}\left(p_{i}(k)\right), \quad p_{i}(k) \in P_{i}(k) ;$

until $j=N c$

$m \leftarrow 1$;

repeat

$p_{i}^{B r}(k) \leftarrow T_{r}^{B}\left(p_{i}^{B c}(k)\right), \quad p_{i}^{B c}(k) \in P_{i}^{B c}(k) ;$

until $m=N r e$

$A_{i}(k) \leftarrow P_{i}^{B r}(k)$;

$a_{i}^{C c}(k) \leftarrow T_{c}^{C}\left(a_{i}(k)\right), \quad a_{i}(k) \in A_{i}(k) ;$

$a_{i}^{C g}(k) \leftarrow T_{g}^{C}\left(a_{i}^{C c}(k)\right), \quad a_{i}^{C c}(k) \in A_{i}^{C c}(k)$;

$a_{i}^{C s}(k) \leftarrow T_{s}^{C}\left(a_{i}^{C g}(k)\right), \quad a_{i}^{C g}(k) \in A_{i}^{C g}(k)$;

$l \leftarrow 1$;

repeat

If $($ rand $<P e$ ) then

\{

$$
p_{i}^{B e}(k) \leftarrow T_{e}^{B}\left(a_{i}^{C s}(k)\right) ; \quad a_{i}^{C s}(k) \in A_{i}^{C s}(k) ;
$$

\}

until $l=N e d$

$P_{i}(k+1) \leftarrow P_{i}^{B e}(k)$;

$i \leftarrow i+1$

until $i=n$

$k \leftarrow k+1$;

end

end while

IV. Function Optimization and Analysis

In order to verify the optimization performance of the proposed BFA-PSA, six functions, namely, Rosenbrock function $f_{1}$, Branins's rcos function $f_{2}$, Goldstein-Price's function $f_{3}$, Himmelbaut function $f_{4}$, Rastrigrin function $f_{5}$, Schaffer 's function $f_{6}$ are tested using Matlab7.01 on PIV $2.99 \mathrm{GHz} 2 \mathrm{~GB}$ memory computer, and the test results are compared with the testing results of GA and CSA. Considering the randomness of three intelligent algorithms (i.e. BFA-PSA, GA and CSA [8]), each function is tested 
independently for 50 times.

1. Rosenbrock function

$\min f_{1}=100\left(x_{2}-x_{1}^{2}\right)^{2}+\left(1-x_{1}\right)^{2}$

Where, $x_{i} \in[-5.12,5.12], 1 \leq i \leq 2, f^{*}=0$.

2. Branins's rcos function

$\min f_{2}=\left(y-5.1 /\left(4 \times \pi^{2}\right) \times x^{2}+5 / \pi \times x-6\right)^{2}+10 \times(1-1 /(8 \times \pi)) \times \cos (x)+10$

Where, $x_{1} \in[-5,10], x_{2} \in[0,15], f^{*}=0.397887$.

3. Goldstein-Price's function

$$
\begin{aligned}
\min f_{3}= & \left(1+\left(x_{1}+x_{2}+1\right)^{2}\left(19-14 x_{1}+3 x_{1}^{2}-14 x_{2}+6 x_{1} x_{2}+3\left(x_{2}^{2}\right)\right)\right) \\
& \left(30+\left(\left(2 x_{1}-3 x_{2}\right)^{2}\right)\left(18-32 x_{1}+12 x_{1}^{2}+48 x_{2}-36 x_{1} x_{2}+27 x_{2}^{2}\right)\right)
\end{aligned}
$$

Where, $x_{i} \in[-2,2], 1 \leq i \leq 2, f^{*}=3$.

4. Himmelbaut function

$$
\min f_{4}=\left(x_{1}^{2}+x_{2}+11\right)^{2}+\left(x_{1}+x_{2}^{2}-7\right)^{2}
$$

Where, $x_{i} \in[-6,6], 1 \leq i \leq 2, f^{*}=0$.

5. Rastrigrin function

$$
\min f_{5}=20+x_{1}^{2}-10 \cos \left(2 \pi x_{1}\right)+x_{2}^{2}-10 \cos \left(2 \pi x_{2}\right)
$$

Where, $x_{i} \in[-5.12,5.12], 1 \leq i \leq 2, f^{*}=0$.

6. Schaffer's Function

\begin{tabular}{|c|c|c|c|c|c|c|c|c|c|c|c|c|c|}
\hline \multirow[t]{2}{*}{ Function } & \multirow[t]{2}{*}{ Precision } & \multicolumn{3}{|c|}{$\begin{array}{l}\text { Number of the } \\
\text { optimal solution }\end{array}$} & \multicolumn{3}{|c|}{$\begin{array}{l}\text { Maximum } \\
\text { convergence } \\
\text { generation }\end{array}$} & \multicolumn{3}{|c|}{$\begin{array}{c}\text { Average } \\
\text { convergence } \\
\text { generation }\end{array}$} & \multicolumn{3}{|c|}{ Stand deviation } \\
\hline & & GA & CSA & $\begin{array}{l}\text { BFA- } \\
\text { PSA }\end{array}$ & GA & CSA & $\begin{array}{l}\text { BFA- } \\
\text { PSA }\end{array}$ & GA & CSA & $\begin{array}{l}\text { BFA- } \\
\text { PSA }\end{array}$ & GA & CSA & $\begin{array}{c}\text { BFA- } \\
\text { PSA }\end{array}$ \\
\hline$f_{1}$ & $10^{-3}$ & 15 & 21 & 25 & 775 & 95 & 41 & 90.30 & 13.98 & 5.78 & 321.33 & 33.33 & 12.04 \\
\hline$f_{2}$ & $10^{-3}$ & 39 & 50 & 50 & 78 & 90 & 70 & 27.16 & 25.68 & 4.89 & 22.91 & 20.26 & 9.87 \\
\hline$f_{3}$ & $10^{-3}$ & 50 & 50 & 50 & 54 & 25 & 17 & 36.10 & 9.50 & 6.14 & 20.93 & 4.63 & 2.44 \\
\hline$f_{4}$ & $10^{-3}$ & 43 & 50 & 50 & 934 & 90 & 54 & 83.91 & 30.36 & 7.34 & 152.82 & 24.25 & 9.87 \\
\hline$f_{5}$ & $10^{-5}$ & 50 & 50 & 50 & 251 & 97 & 12 & 83.00 & 42.85 & 5.26 & 46.97 & 22.12 & 1.47 \\
\hline$f_{6}$ & $10^{-5}$ & 26 & 29 & 30 & 917 & 98 & 77 & 339.81 & 54.97 & 17.83 & 291.32 & 26.04 & 17.99 \\
\hline
\end{tabular}

$$
\begin{aligned}
& \min f_{6}=0.5+\left(\left(\sin \left(\operatorname{sqrt}\left(x_{1}^{2}+x_{2}^{2}\right)\right)\right)^{2}-0.5\right) /\left(\left(1+0.0001\left(x_{1}^{2}+x_{2}^{2}\right)^{2}\right)^{2}\right) \\
& \text { Where, } x_{i} \in[-10,10], 1 \leq i \leq 2, f^{*}=0 .
\end{aligned}
$$

Table 1 Function optimization results of GA, CSA and BFA

Table 1 gives the optimization results of three optimization algorithms. From the table, it can be seen that the proposed the number of the optimal solution of BFA-PSA in six function optimizations are the most, which shows the strong optimization ability of the BFA-PSA. The maximum convergence generation and average convergence generation shows the quick convergence speed. The stand deviation shows the good stability. All good optimization performances of the proposed BFA-PSA are mainly due to the introduction of the BFA in the PSA. The convergence speed of the individuals is improved because the chemotaxis operator and reproduction operator are introduced into the clonal selection algorithm. Further more, because the eliminationdispersal operator is added in the clonal selection algorithm, the individuals will die according to certain probability $\mathrm{p}$ and new individuals will replace them, which retains the diversity of the population well.

Fig. 3 shows the evolutionary curves of GA, CGA and BFA-PSA during the function optimization. From the figure, it can be seen that compared with GA, CGA, the convergence speed of BFA-PSA is faster and the global optimization ability is stronger, which further verify the effectiveness of the BFA-PSA. 


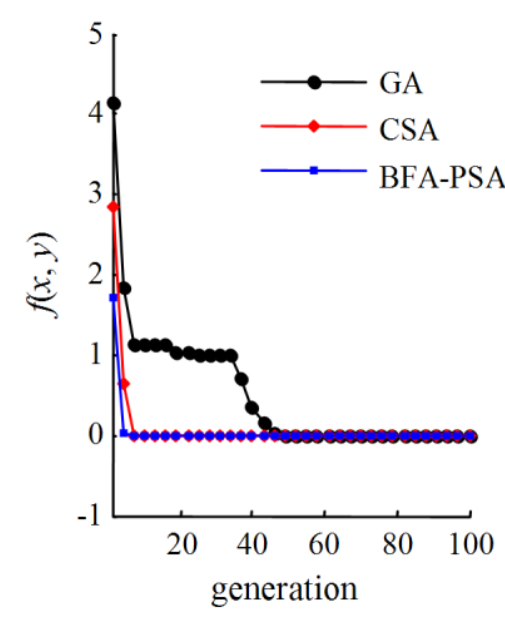

(a)The average solution

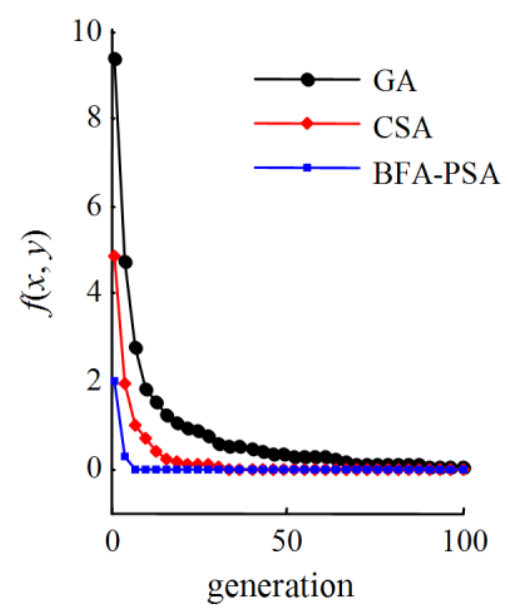

(b) The optimal solution

Fig. 3 Average evolution curves of Schaffer 's function

\section{Optimization of the PID Control Parameters for the Brushless DC Motor}

In order to further verify the validity of the proposed BFA-PSA in the practical application, the BFAPSA is applied to the optimization of the PID control parameters for the brushless DC Motor.

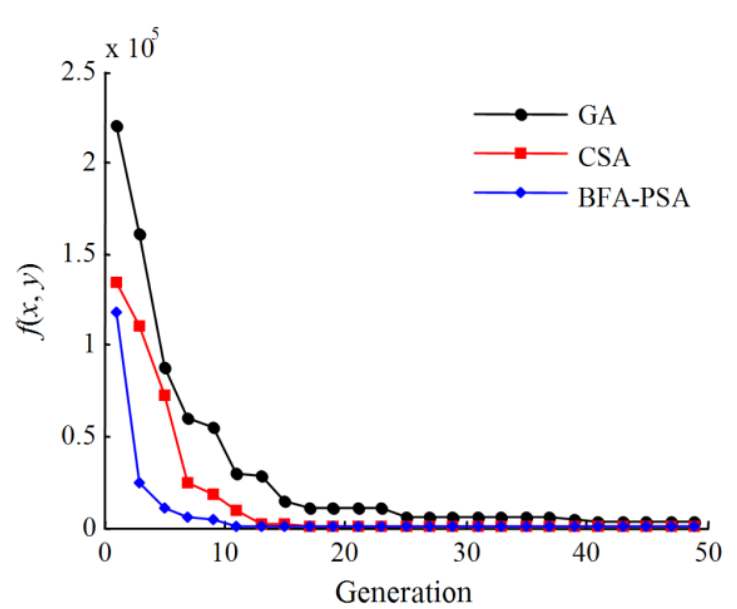

Fig. 4 Convergence curves

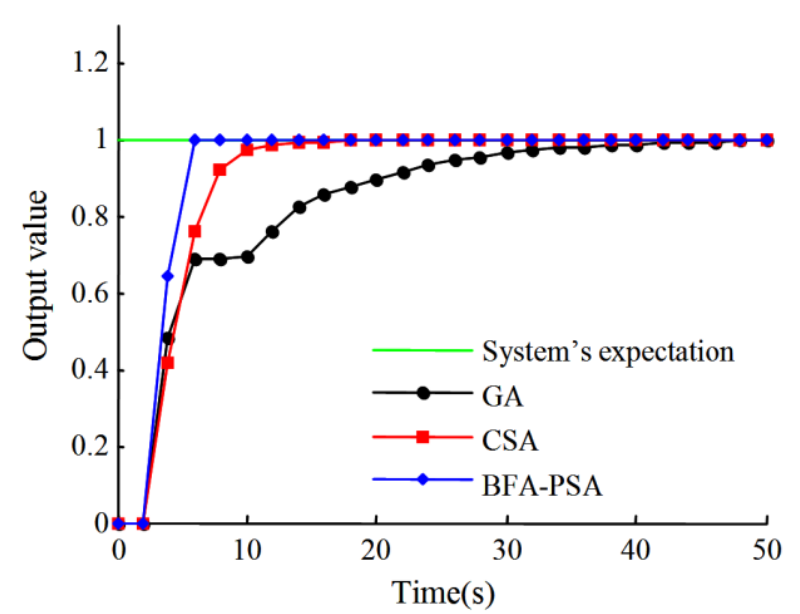

Fig. 5 Unit step response curve

Fig. 4 gives the convergence curves of the three intelligent algorithms. From the figure, it can be seen that the convergence speed of the BFA-PSA is still the quickest.

Fig. 5 gives the unit step response curve. From the figure, we can see that the response speed of unit step based on the PID whose parameters are optimized by the proposed BFA-PSA is also the quickest.

The convergence curves in the optimization of PID parameters and the unit step response all verify the validity and superiority of the BFA-PSA.

\section{Conclusion}

In order to solve the optimization of PID control parameters for the brushless DC motor, a bacterial foraging algorithm-based polyclonal selection optimization algorithm (BFA-PSA) is proposed. Three operators of bacterial foraging algorithm, namely chemotaxis operator, reproduction operator and elimination-dispersal operator, are added in the polyclonal selection algorithm to improve the convergence speed and retain the diversity of the population. The optimization results of functions and PID control parameters verify the validity and superiority of the proposed BFA-PSA.

\section{References}

[1] J.j. Zhang, Optimization parameters of PID controller parameters based on particle swarm optimization, Computer Simulation, 27(10), 2010, 191-193.

[2] H.P. Yin, Y. Chai, Parameters optimization design of PID controller based on ant colony algorithms, Computer Engineering and Applications, 43(17),2007, 4-7.

[3] L.Y. Yu, S.M. Jiao, PID optimization based on fish swarm algorithm, Computer Simulation, 31(3), 2014, 155-158. 
[4] D.F. Wang, P. Han, Variable arguments PID control for main steam temperature system based on immune genetic optimization, Proceedings of the CSEE, 23(9) ,2003, 212-217.

[5] G.Q. Han, P. Wang, N.H. Qin, PID control of brushless DC motor based on genetic algorithm, Science Mosaic,6,2010,135-137.

[6] L. Cong, Y.H. Sha, L.C. Jiao, Application of immune clone selection algorithm to image segmentation, Journal of Electronics \& Information Technology,28(7),2006,1169-1173.

[7] Y.L. Zhou, Research and application on bacterial foraging optimization algorithm, Computer and Applications, 46(20), 2010,16-21.

[8] M.X. Yuan, H.F. Du, S.A. Wang, J. Zhuang, Chaos small-world optimal algorithm based on population entropy, Journal of Xi'an Jiaotong University, 42(9), 2008, 1137-1141. 